\title{
Amplatzer vascular plug in renal artery embolization: case report and review of the literature
}

\author{
Antonio Catelli $i^{*}$, Giovanni Loiudice ${ }^{1}$, Antonio Corvino², Umberto Marcello Bracale ${ }^{3}$, Mario Quarantelli \\ and Pietro Venetucci ${ }^{1}$
}

\begin{abstract}
Background: Arterial embolization constitutes a safe and effective therapeutic possibility in the treatment of numerous renal pathologies in election and in urgency. Over the years, numerous embolizing materials have been proposed: fibrin (temporary occlusion), glues, coils, and microspheres. The use of St. Jude Amplatzer vascular plug (AVP) constitutes a more recent and less widespread therapeutic choice, and its use in the renal district is not yet widespread although potentially very valid.

Case presentation: We present 3 renal arterial embolizations performed with AVP performed between January 2019 and February 2020: two patients had a very bulky renal heteroplastic lesion and were candidates for nephrectomy, and a third patient instead was affected by a high-flow FAV post-biopsy. In our experience, the use of AVP for the treatment of renal arteriovenous fistula and neoplastic masses has given excellent results without any complications during and after the treatment. In all cases, a single plug was used which perfectly adapted to the target artery resulting in complete occlusion of the vessel in a short time.

Conclusion: Renal artery embolization performed with AVP has growing potential and numerous advantages in terms of the time of the procedure, speed of embolization, and precision of occlusion, but with a relative increase in costs.
\end{abstract}

Keywords: Embolization, Interventional radiology, Coils, Plug, Case report

\section{Background}

Renal artery embolization is an interventional radiology procedure that over the years has proven to be safe and effective in the treatment of numerous pathologies both in election and in urgency: active post-traumatic or post-biopsy bleeding, kidney tumors, arteriovenous fistula (FAV), and vascular malformation [1]. Over the years, numerous embolizing materials have been proposed: fibrin (temporary occlusion), glues, coils, and microspheres [1]. The plugs represent a vascular occlusion system initially used at the cardiological pathologies (foramen ovale or DIA occlusion) and extended in the peripheral area with the necessary structural changes. Since 2004, it has been approved for the clinical use of AVP exclusively dedicated to peripheral vascular structures. Its use in the renal district is not yet widespread although potentially very valid. We present 3 renal arterial embolizations performed with AVP performed between January 2019 and February 2020: two patients had a very bulky renal heteroplastic lesion and were candidates for nephrectomy, and a third patient instead was affected by a high-flow FAV post-biopsy.

\footnotetext{
* Correspondence: catelliantonio89@gmail.com

'Advanced Biomedical Sciences Department, University Federico II of Naples (UNINA), via S. Pansini 5, I-80131 Naples, Italy

Full list of author information is available at the end of the article
}

\section{Springer Open}

(- The Author(s). 2020 Open Access This article is licensed under a Creative Commons Attribution 4.0 International License, which permits use, sharing, adaptation, distribution and reproduction in any medium or format, as long as you give appropriate credit to the original author(s) and the source, provide a link to the Creative Commons licence, and indicate if changes were made. The images or other third party material in this article are included in the article's Creative Commons licence, unless indicated otherwise in a credit line to the material. If material is not included in the article's Creative Commons licence and your intended use is not permitted by statutory regulation or exceeds the permitted use, you will need to obtain permission directly from the copyright holder. To view a copy of this licence, visit http://creativecommons.org/licenses/by/4.0/. 


\section{Case presentation}

\section{First patient}

A 40-year-old man patient underwent ultrasound examination for macro-hematuria. The examination revealed the presence of a massive right renal mass. The patient was hospitalized in the general surgery department. On clinical examination, the abdomen was moderately treatable. Blood pressure was 140/80 mmHg. The laboratory values of $\mathrm{Hb}$ were $12.0 \mathrm{~g} / \mathrm{dL}$. Liver, kidney, and coagulation function values were normal. Tumor markers were negative. Lung and heart functions were regular. He therefore performed an abdominal angio-CT exam and a subsequent micro-histological biopsy at our interventional radiology department which confirmed the presence of a solid neoplastic mass of $30 \times 20 \mathrm{~cm}$ in the right kidney. It was remarkably vascularized and determined a total subversion of the renal parenchymal architecture and a significant regional local mass effect on the adjacent abdominal structures. There were no lymphadenopathies, ascites, and metastases. The upper urinary tract and ipsilateral adrenal gland were completely incorporated into the carcinoma. The neoplasm was mainly vascularized both by a large efferent branch of the renal artery and by the lower adrenal artery, also of a significantly increased size. In agreement with the surgeon, it was decided to perform a selective embolization of the right renal artery and then proceed to a total nephrectomy within the following $72 \mathrm{~h}$. After local anesthesia and right common femoral access with a 5-Fr introducer, the right renal artery was selectively catheterized. Diagnostic angiography was performed which confirmed the vascular afferents highlighted in the angio-CT exam. Therefore, a 6-Fr-long armed introducer with the distal end in the renal artery was positioned and AVP II of 16 $\times 12 \mathrm{~mm}$ was released. After $10 \mathrm{~min}$, angiography was performed which showed the absence of the flow downstream of the AVP. The procedure was completed with mechanical hemostasis of the access site. The patient developed post-embolization ischemic syndrome managed with symptomatic drugs. The total mass exeresis surgery was performed in the $6 \mathrm{~h}$ following the procedure, during which no blood transfusions were performed. The excised expansive formation had a total weight of $7 \mathrm{~kg}$. Cardio-respiratory complications were not observed.

\section{Second patient}

A 60-year-old woman patient was hospitalized in the general surgery department for the treatment of a large right renal neoplastic mass associated with intra-tumor fistula. On clinical examination, the abdomen was treatable. The blood count showed moderate anemia: $8 \mathrm{~g} / \mathrm{dL}$. Microhematuria was detected. Markers of liver function, kidney function, and coagulation indices were normal. Blood pressure was $125 / 70 \mathrm{mmHg}$. Lung and heart functions were regular. Angio-CT exam was performed. The examination confirmed the presence of a heteroplastic solid hypodense mass of $35 \times 20 \mathrm{~mm}$, hypervascularized. Ascites, lymphadenopathy, and metastasis were not evident. There was an absence of hydronephrosis. At angio-CT control, a single hypertrophic renal artery was detected, with intra-tumor fistula. The surgeon indicated excision surgery and required pre-operative embolization to reduce the bleeding risk. It was performed after local anesthesia with right common femoral access and selective distal catheterization of the right renal artery with a long-armed introducer. The subsequent angiography confirmed the presence of the neoplasm with contextual fistula and then proceeded to the positioning and release of AVP II of $16 \times 12 \mathrm{~mm}$ at the distal bifurcation of the vessel. At 10-min angiography, complete occlusion of the intra-parenchymal arteries and disappearance of the arteriovenous fistula were confirmed. The patient was operated on in the following 24 $\mathrm{h}$; during the surgery, no blood transfusion was performed. The patient did not develop cardio-respiratory complications.

\section{Third patient}

A 57-year-old woman patient in follow-up for a nephrotic syndrome about 10 years in the nephrology department. The patient developed left arteriovenous fistula after a kidney biopsy performed 5 years earlier. Although the nephrotic syndrome was well compensated, the patient had been showing signs of heart failure for about 2 years, which had worsened in the past 6 months. Interventional radiology consultancy was performed with indication of embolization of the renal fistula. Hemoglobin, liver function, and coagulation indices are in the norm. Preliminary abdominal angio-CT examination confirmed the presence of a high-flow fistula at the meso-renal site. After anesthesia, selective catheterization of the left renal artery with a 5-Fr catheter was performed through 5-Fr right common femoral access; the angiography confirmed high-flow fistula at the meso-renal site. AVP IV $7 \times 12 \mathrm{~mm}$ was released via the 5 -Fr catheter at the site. At the check after 10 min, the fistula did not show flow with hypoperfusion < $20 \%$ of the renal parenchyma. The patient developed a post-embolization syndrome characterized by pain and fever that was resolved with medical therapy. No complications were found. An Eco color Doppler exam was performed immediately after the procedure, and after 30 days and 3 months, which confirmed the absence of the FAV. At 6 months, angio-CT was performed which showed no flow in the site of the treated arteriovenous fistula. The signs of heart failure decreased significantly after about 6 months after the surgery. 


\section{Discussion}

\section{Paragraph 1}

The AVP is a device designed and produced by St. Jude since 2004 for the embolization of peripheral arterial and venous vessels of various sizes. Over the years, several models have been designed that have progressively implemented the therapeutic possibilities of this family of devices. Currently, there are 4 models that share two common components: a vascular plug and a delivery wire. In addition, all devices have a self-expanding system, a platinum radiopaque marker, and a controlled and fast release mechanism. The structural characteristics of the different types of AVP are as follows: AVP I has a single lobe and single layer, AVP II has 3 lobes and multilayer, AVP III is bilobed and multilayer, and AVP IV is bilobed and single layer [2]. The indications are different: AVP I is indicated for vessels that have a reduced landing area, AVP II is very ductile due to the variability of available calibers and is indicated when rapid occlusion of the flow is required; contraindicated when there is a poor "landing zone", the AVP III is used in medium-sized vessels, with a tortuous course and which have a high flow. AVP IV, available from 2009, with sizes from 4 to $8 \mathrm{~mm}$ represents the recent solution of the AVP family. The device can be released through a diagnostic 5-Fr catheter with compatible guide 0.038, and the structural characteristics allow both the release in irregular course vessels and a rapid occlusion of the target $[2,3]$. The literature is very vast regarding the use of the different types of devices of the AVP family in various treatments such as in FAV, aneurysms, portal vein embolization, and post EVAR endoleak. Furthermore, cases of occlusions of splanchnic and bronchial branches as well as in the treatment of superficial FAV in hemodialysis are also described. Although there are a small number of scientific works that describe the release of AVP in the embolization of the renal vessels, their use is becoming increasingly widespread and routine $[2,4,5]$. The embolization of the renal arteries represents the gold standard for acute bleeding; it is a valid support for the treatment of arteriovenous fistula and for the therapeutic management of large hypervascularized renal heteroplastic masses but it is strongly recommended also in cases of vascular malformations [1-6]. In pre-operative planning, knowledge of normal anatomy and renal artery variants found in $30 \%$ of the population is fundamental. In addition to the knowledge of the anatomy of the vessels, it is essential to have information on the values of hemoglobin; hematocrit; renal, cardiac, and hepatic function; and coagulation indices [4]. Renal venous arterial malformations are even rarer and can benefit from an endovascular treatment which, however, as with MAV from other districts, is not always decisive, frequently requiring more sessions. The embolizing

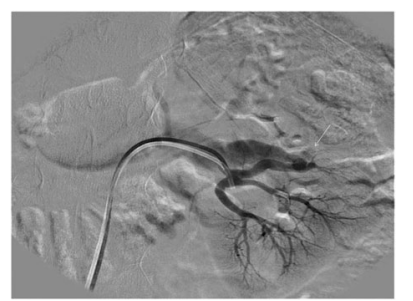

Fig. 1 AVF treatment. High-flow fistula angiography (arrow)

material to be used is chosen based on the size, course, and flow of the vessel. The use, even combined, of embolizing materials such as fibrin, PVA, EVOH, and microcoils is mainly indicated for the embolization of peripheral distal vessels of small caliber. They are widely used in case of distal pseudoaneurysms or bleeding. The coils and plugs are mainly indicated for the occlusion of medium-proximal vessels. The plugs, although still not widespread in the clinical setting, have a precise release system as well as a low risk of migration; often, a single plug is required for a complete occlusion.

\section{Paragraph 2}

Indications for embolization in the therapeutic management of neoplastic renal masses include palliative treatments for mass reduction and hemorrhagic risk in patients candidates for total or partial nephrectomy or radio-ablation treatments as well as for control of hematuria and/or active intra- and peri-injury bleeding. Non-traumatic kidney bleeding is a rare event, but it can be very life-threatening. It is a very frequent event in large benign or malignant neoplasms (61\%); among these, it is very frequent in angiomyolipoma (29\%) and renal cell carcinoma $(26 \%)[7,8]$. The ischemia produced by the pre-operative endovascular procedure also involves the formation of a clearer edematous cleavage plan between the mass and the nearby tissues allowing the surgeon a more rapid and precise removal; this condition is more pronounced especially within $72 \mathrm{~h}$ of the procedure. The ischemic syndrome can be of various entities mainly characterized by leukocytosis, nausea, vomiting, and fever $[9,10]$. However, a risk-benefit assessment is essential before each procedure $[9,10]$.

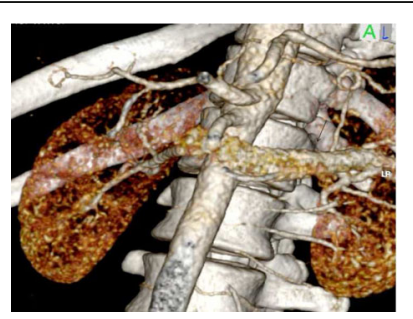

Fig. 2 AVF treatment. Angio-CT 3D V. R. reconstruction (arrow) 


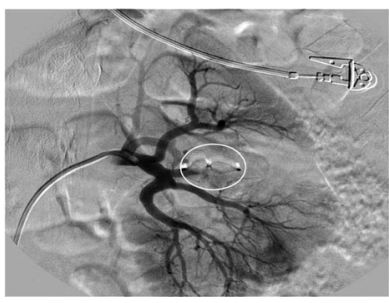

Fig. 3 AVF treatment. Subsequent treatment with the release of AVP IV (circle) at the corresponding afferent arterial branch and flow stop

\section{Paragraph 3}

Kidney biopsy is a minimally invasive method. However, it can give major and minor complications. Minor complications are hematuria, small peri-renal hematomas, arteriovenous fistulas, and pain that resolves spontaneously. The major complications related to massive bleeding with hemodynamic instability, high-flow arteriovenous fistulas, macro-ematura, and voluminous peri-renal hematomas. Bleeding is the most frequent complication. It occurs in $12-24 \mathrm{~h}$ after the biopsy. The literature remarks variable complication rates, from 5 to $16 \%$ of cases including $9-13 \%$ of hematuria. Blood transfusion is essential in $0.1-3 \%$ of cases $[11,12]$. Several studies have reported incidences of complications of 7.4-11\% after kidney biopsy. Renal arteriovenous fistulas represent a pathological condition characterized by an abnormal connection between an arterial and venous vessel. They are classified according to etiology into idiopathic, acquired, and congenital. Idiopathic and congenital forms represent $30 \%$ of all renal fistulas [13, 14]. Acquired fistula can be secondary to traumatic events, carcinoma, arteritis, and biopsy [13, 14]. Most renal arteriovenous fistulas due to renal biopsy are asymptomatic and resolve spontaneously within 2 years, but some may be symptomatic and require interventional treatment [15]. The treatment has been surgical for years with a partial or total nephrectomy and ligation of the arterial vessel $[13,14,16]$. Endovascular treatment allows to occlude the afferent vessel and has progressively replaced surgery therapy.

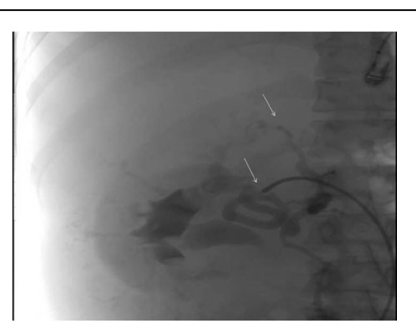

Fig. 4 Neoplastic mass treatment. Angiography shows that the neoplasm is mainly vascularized by both a large efferent branch of the renal artery (arrow) and the lower adrenal artery (arrow)

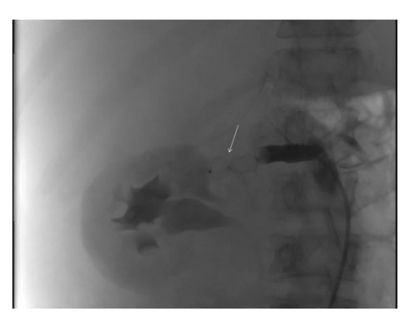

Fig. 5 Neoplastic mass treatment. Subsequent treatment with the release of AVP II (arrow) occluding the renal artery at the origin of the adrenal artery and flow stop

\section{Conclusion}

In our experience, the use of AVP for the treatment of renal arteriovenous fistula and neoplastic masses has given excellent results without any complications during and after the treatment (Figs. 1, 2, 3, 4, 5, 6 and 7). In all cases, a single plug was used which perfectly adapted to the target artery resulting in complete occlusion of the vessel in a short time. Furthermore, the use of the single device has reduced the procedural times with respect to the positioning of coils; very often, more spirals are needed for the complete occlusion of the vessel. Although the cost of the single AVP is higher than the single spiral, often, the use of more coils and above all of the technologically more advanced ones (with 3D- or $4 \mathrm{D}$-controlled release) involves an equal or higher cost. The technical characteristics of the device allowed very precise preliminary checks that ensured a selective occlusion of the target vascular segment with a very low risk of migration. The release of the plug occurred quickly and the complete occlusion obtained in $10 \mathrm{~min}$. The structural characteristics of the device determine a complete embolization within 10-15 min from the release in the vessel since it progressively adapts to the target branch causing a progressive slowing down of the blood flow until its complete stop. The release system is quick and safe. In conclusion, the use of AVP for renal artery embolization for the treatment of renal fistula and neoplastic mass has shown an excellent result without finding any complications during and after the interventional procedure. The use of a single device has always

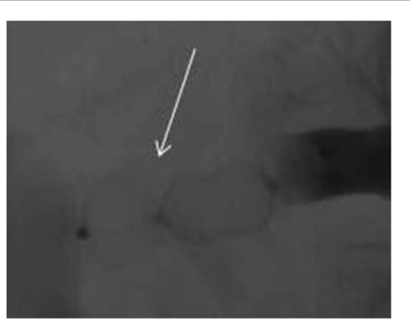

Fig. 6 The structural characteristics of the AVP II that is composed of 3 lobes and a multilayer 


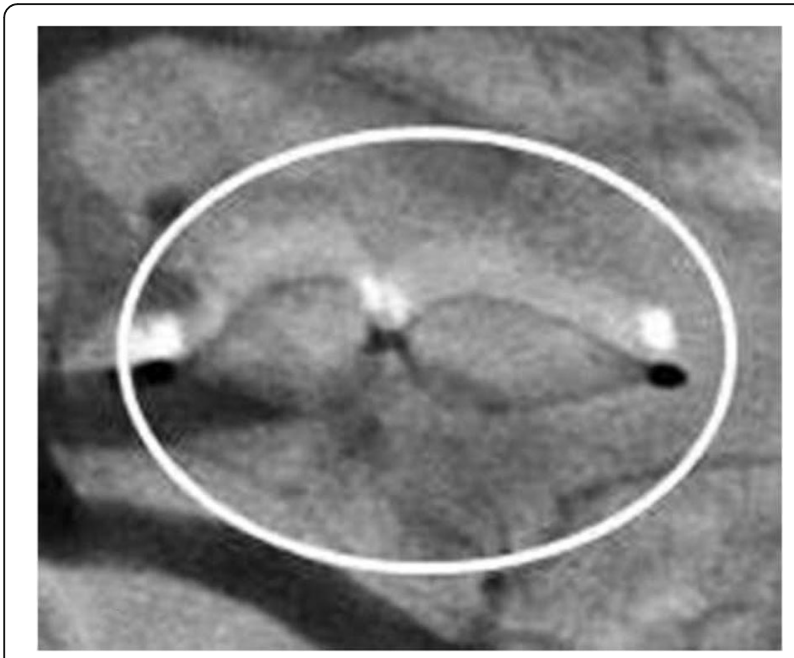

Fig. 7 The structural characteristics of the AVP IV that is composed 2 lobes and a single layer

been necessary. Migration was not observed. Full embolization was observed within $10 \mathrm{~min}$ in all procedures. During the surgery of the two patients with large tumor mass, no blood transfusions were necessary. ECD exams and CT performed 6 months after fistula treatment did not show recanalization with regular opacification of the remaining vascular angioarchitecture. The area of infarction appeared on CT slightly reduced in volume with clearer margins for post-embolization adaptation. Currently, all patients are in good condition and perform follow-up in their referral departments.

\section{Abbreviations}

AVP: St. Jude Amplatzer vascular plug; AVP II: St. Jude Amplatzer vascular plug type II; AVP IV: St. Jude Amplatzer vascular plug type IV; FAV: Renal arteriovenous fistula

\section{Acknowledgements}

Not applicable.

\section{Authors' contributions}

A.Cat. and P.V. are the main authors who collected the data and wrote the manuscript. A.Cat. sent to the newspaper. The authors have read and approved the final manuscript.

\section{Funding}

Not applicable.

\section{Availability of data and materials}

All the data and materials were obtained from patients registered in our hospital.

\section{Ethics approval and consent to participate}

Patients included in this study gave written consent to participate in this research.

\section{Consent for publication}

All participants gave written consent to the publication of the manuscript. The images presented do not compromise the patient's anonymity.

\section{Competing interests}

The authors declare that they have no competing interests.

\section{Author details}

Advanced Biomedical Sciences Department, University Federico II of Naples (UNINA), via S. Pansini 5, I-80131 Naples, Italy. ${ }^{2}$ Motor Science and Wellness Department, University of Naples "Parthenope", via F. Acton 38, I-80133 Naples, Italy. ${ }^{3}$ Public Hearth Department, University of Naples "Parthenope", via F. Acton 38, I-80133 Naples, Italy.

Received: 28 May 2020 Accepted: 4 August 2020

Published online: 18 August 2020

\section{References}

1. Jardinet $T$, Bonne L, Oyen R, Maleux G (2020) Initial experience with the microvascular plug in selective renal artery embolization. Vasc Endovasc Surg 54(3):240-246. https://doi.org/10.1177/1538574419897500

2. Ramakrishnan S (2015) Vascular plugs - a key companion to interventionists - 'just plug it'. Indian Heart J 67(4):399-405. https://doi.org/10.1016/j.ihj. 2015.07.001

3. Wang W, Li H, Tam MD, Zhou D, Wang DX, Spain J (2012) The Amplatzer vascular plug: a review of the device and its clinical applications. Cardiovasc Intervent Radiol 35(4):725-740. https://doi.org/10.1007/s00270-012-0387-z

4. Lopera JE (2015) The Amplatzer vascular plug: review of evolution and current applications. Semin Interv Radiol 32(4):356-369. https://doi.org/10. 1055/s-0035-1564810

5. Güneyli S, Cs Inar C, Bozkaya H, Parıldar M, Oran I. (2014). Applications of the Amplatzer Vascular Plug to various vascular lesions. Diagn Interv Radiol.;20: $155 \mathrm{e} 159$

6. Ramaswamy RS, Darcy MD (2016) Arterial embolization for the treatment of renal masses and traumatic renal injuries. Tech Vasc Interv Radiol 19(3):203210. https://doi.org/10.1053/j.tvir.2016.06.005

7. Zhang JQ, Fielding JR, Zou KH (2002) Etiology of spontaneous perirenal hemorrhage: a meta-analysis. J Urol 167:1593-1596

8. Durak D, Eren F, Inanir NT (2014) Spontaneous rupture of a renal cell carcinoma associated with fatal bleeding. Maedica (Buchar) 9(3):275-277

9. Venetucci P, Quarantelli M, laccarino V $(2011,2011)$ A rare case of recurrent hematuria from right kidney: radiologic diagnosis and treatment. ISRN Urol: 159104. https://doi.org/10.5402/2011/159104

10. Patatas K, Robinson GJ, Ettles DF (2013) Patterns of renal angio-myolipoma regression post embolisation on medium- to long-term follow-up. Br J Radiol 86:20120633

11. Choi IJ, Jeong HJ, Han DS (2001) An analysis of 4.514 cases of renal biopsies in Korea. Yonsei Med J 42(2):247-254. https://doi.org/10.3349/ymj.2001.42.2.247

12. Burstein D, Korbet S, Schwartz M (1993) The use of the automated core biopsy system in percutaneous renal biopsies. A comparative study. Am J Kidney Dis 22:545-552

13. Abdel-Aal, Elsabbagh A, Soliman H, Hamed M, Underwood E, Saddekni S (2014) Percutaneous embolization of a postnephrectomy arteriovenous fistula with intervening pseudoaneurysm using the Amplatzer vascular plug 2. Vasc Endovasc Surg 48(7-8):516-521. https://doi.org/10.1177/ 1538574414561230

14. Osawa T, Watarai Y, Morita K, Kakizaki H, Nonomura K (2006) Surgery for giant high-flow renal arteriovenous fistula: experience in one institution. BJU Int 97(4):794-798

15. Van den Broecke $M$, Vereecke $E$, de Visschere P (2020) Renal arteriovenous fistula. J Belg Soc Radiol 104(1):10. https://doi.org/10.5334/jbsr.2032

16. Haochen W, Jian W, Li S, Tianshi L, Xiaogiang T, Yinghua Z (2019) Superselective renal artery embolization for bleeding complications after percutaneous renal biopsy: a single-center experience. J Int Med Res 47(4): 1649-1659. https://doi.org/10.1177/0300060519828528

\section{Publisher's Note}

Springer Nature remains neutral with regard to jurisdictional claims in published maps and institutional affiliations. 